\title{
Star formation in NGC 3603
}

\author{
Mauricio Tapia and Brenda Pérez \\ Instituto de Astronomía, UNAM, Ensenada, B.C., Mexico
}

\section{Introduction}

NGC 3603 is the most massive optically visible $\mathrm{H}$ II region in our Galaxy and is considered to be the Galactic analogue to 30 Doradus and, thus, a prototype of the starburst phenomenon. Its core, known as WR 43 (HD 97950), contains several dozens of O-B0 stars within $35^{\prime \prime}$. These have been studied with very high resolution in the optical and near-IR. Melnick, Tapia \& Terlevich (1989) concluded that the starburst cluster has an age spread of 1 to $2 \mathrm{Myr}$ and that its boundaries extend to $\sim 60^{\prime \prime}$, while Eisenhauer et al. (1998) determined that the lower mass stars in the core are younger than $1 \mathrm{Myr}$.

Age stratification in NGC 3603 was first suggested by Tapia (1981), Persi et al. (1985) and Melnick et al. (1989). The oldest generation of stars are the red supergiants located to the north of WR 43, followed by the visible starburst cluster. Frogel et al. (1977) discovered a number of very young massive stellar objects some $1^{\prime}$ to the west and south of WR 43. Tapia (1981), Persi et al. (1985) and Roth et al. (1986) reported the near-IR photometric and spectroscopic properties of most of these objects.

\section{Observations}

$J H K$ broad-band images were obtained with infrared NICMOS $256 \times 256$-pixel detectors attached to the Las Campanas Observatory 2.5m telescope and the CTIO $1.5 \mathrm{~m}$ telescope. The former included only the central $1^{\prime}$, while the latter covered some $4^{\prime} \times 4^{\prime}$, which included the region of peak emission of ${ }^{12} \mathrm{CO}$. Fig. 1 displays the $K$-band image. $J H K$ photometry of several hundred sources was obtained from the images using DAOPHOT. $H-K$ vs. $J-H$ is also shown.

\section{Results and discussion}

The main results from the analysis of the images and the two-colour and colourmagnitude diagrams presented in this work are the following:

(a) A population of evolved massive red ( $\mathrm{G}, \mathrm{K}$ and $\mathrm{M}$ ) supergiants (older than $5 \mathrm{Myr}$ ), all located $1^{\prime}$ to $3^{\prime}$ to the north of WR 43 , seems to be the remnant of the first generation of massive stars formed in the region.

(b) The compact cluster of more than 50 OB stars, WR 43, is the result of the main episode of star-formation burst, spanning over 0.5 to $2 \mathrm{Myr}$. The huge stellar winds from the cluster have created a dust and gas free expanding bubble which interacts with denser material, marked by the brightest nebulous parts 

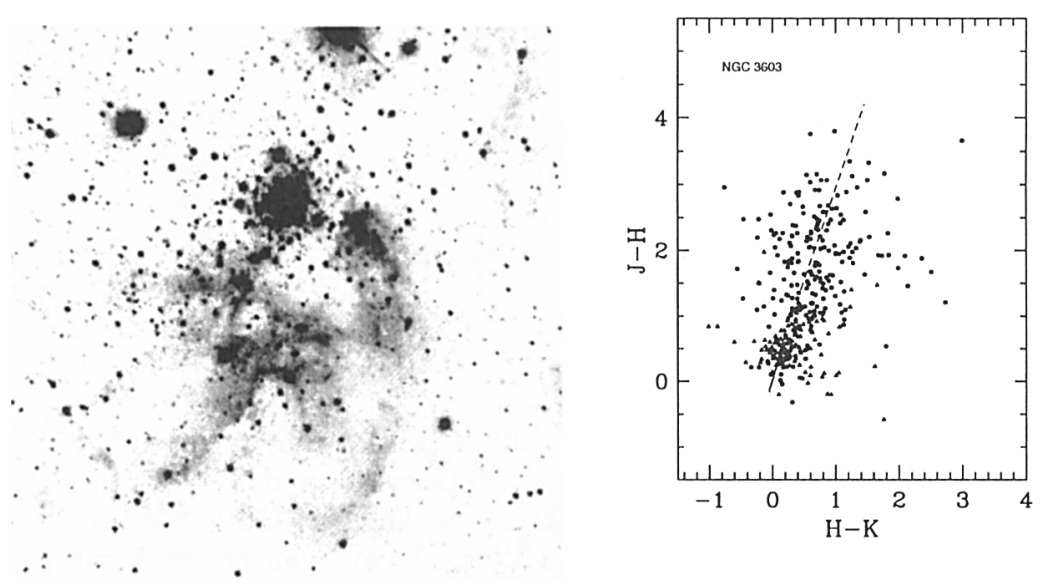

Figure 1. $K$-band image of NGC 3603 and two colour diagram

of NGC 3603 to the W and S. In the near-IR these regions are characterized by bright molecular hydrogen emission.

(c) The most recent star-formation events are present some $2^{\prime} \mathrm{s}$ and $\mathrm{SW}$ of WR 43. A number of medium and high mass very young stellar objects, including a compact embedded cluster, is reported in this work. These are within the boundaries of the near and mid-infrared extended sources IRS-1, IRS-2, IRS-3 and IRS-9 discovered by Frogel et al. (1977) and further analysed by Tapia (1981) and Persi et al. (1985). The most highly reddened objects lie within the region of the peak $\mathrm{CO}$ emission. The ages of these objects are around $0.1 \mathrm{Myr}$ or less. Some of the reddest IR sources are located at the tip of dense dust pillars pointing towards WR 43.

Spatially segregated star-formation sites have recently been reported in detail in 30 Dor (Rubio et al. 1998; Walborn et al. in these Proceedings). As in NGC 3603, this new generation of stars was triggered by the huge starburst which gave birth to $\mathrm{R} 136$. The results presented here further increase the similarities between these giant complexes.

\section{References}

Eisenhauer, F., Quirrenbach, A., Zinnecker, H., Genzel, R. 1998, ApJ 498, 278

Frogel, J.A., Persson, S.E., Aaronson, M. 1977, ApJ 213, 723

Melnick, J., Tapia, M., Terlevich, R. 1989, A\&A 213, 89

Persi, P., Tapia, M., Roth, M., Ferrari-Toniolo, M. 1985, A\&A 144, 275

Roth, M. et al. 1986, in: M. Peimbert \& J. Jugaku (eds.), Star Forming Regions, Proc. IAU Symp. No. 115 (Dordrecht: Reidel), p. 182

Rubio, M., Barbá, R.H, Walborn, N.R., Probst, R.G., Garcia, J., Roth, M.R. 1998, AJ 116,1708

Tapia, M. 1981, PhD Thesis, University of Edinburgh 